\title{
Low Provider Knowledge Is Associated With Less Evidence-Based Lung Cancer Screening
}

\author{
Jennifer A. Lewis, MD, MSa,b; Heidi Chen, $\mathrm{PhD}^{c}$; Kathryn E. Weaver, $\mathrm{PhD}^{\mathrm{d}}$; Lucy B. Spalluto, MD ${ }^{\mathrm{a}, \mathrm{e}}$; \\ Kim L. Sandler, MDe; Leora Horn, MD, MSc ${ }^{b}$; Robert S. Dittus, MD, MPH,f; Pierre P. Massion, MDg,h; \\ Christianne L. Roumie, MD, MPH ${ }^{a, f}$; and Hilary A. Tindle, MD, MPH ${ }^{a, f}$
}

\begin{abstract}
Background: Despite widespread recommendation and supportive policies, screening with low-dose CT (LDCT) is incompletely implemented in the US healthcare system. Low provider knowledge of the lung cancer screening (LCS) guidelines represents a potential barrier to implementation. Therefore, we tested the hypothesis that low provider knowledge of guidelines is associated with less provider-reported screening with LDCT. Patients and Methods: A cross-sectional survey was performed in a large academic medical center and affiliated Veterans Health Administration in the Mid-South United States that comprises hospital and community-based practices. Participants included general medicine providers and specialists who treat patients aged $>50$ years. The primary exposure was LCS guideline knowledge (US Preventive Services Task Force/Centers for Medicare \& Medicaid Services). High knowledge was defined as identifying 3 major screening eligibility criteria $(55$ years as initial age of screening eligibility, smoking status as current or former smoker, and smoking history of $\geq 30$ packyears), and low knowledge was defined as not identifying these 3 criteria. The primary outcome was self-reported LDCT order/referral within the past year, and the secondary outcome was screening chest radiograph. Multivariable logistic regression evaluated the adjusted odds ratio (aOR) of screening by knowledge. Results: Of 625 providers recruited, 407 (65\%) responded, and 378 (60.5\%) were analyzed. Overall, 233 providers (62\%) demonstrated low LCS knowledge, and 224 (59\%) reported ordering/referring for LDCT. The aOR of ordering/referring LDCT was less among providers with low knowledge $(0.41 ; 95 \% \mathrm{Cl}, 0.24-0.71)$ than among those with high knowledge. More providers with low knowledge reported ordering screening chest radiographs (aOR, $2.7 ; 95 \% \mathrm{Cl}, 1.4-5.0$ ) within the past year. Conclusions: Referring provider knowledge of LCS guidelines is low and directly proportional to the ordering rate for LDCT in an at-risk US population. Strategies to advance evidence-based LCS should incorporate provider education and system-level interventions to address gaps in provider knowledge. J Natl Compr Canc Netw 2019;17(4):339-346 doi: $10.6004 /$ jnccn.2018.7101
\end{abstract}

\footnotetext{
${ }^{a}$ Geriatric Research, Education and Clinical Center, Veterans Health Administration - Tennessee Valley Healthcare System, Nashville, Tennessee; ${ }^{b}$ Division of Hematology/Oncology, Department of Medicine, and ${ }^{\mathrm{c} D e p a r t m e n t}$ of Biostatistics, Vanderbilt University Medical Center, Nashville, Tennessee; ${ }^{\mathrm{d} D e p a r t m e n t}$ of Social Sciences and Health Policy, Wake Forest School of Medicine, Winston-Salem, North Carolina; ${ }^{e}$ Department of Radiology, ${ }^{f}$ Division of General Internal Medicine and Public Health, Department of Medicine, and 9Division of Allergy, Pulmonary, and Critical Care Medicine, Vanderbilt University Medical Center, Nashville, Tennessee; and hepartment of Medicine, Veterans
} Health Administration - Tennessee Valley Healthcare System, Nashville, Tennessee.

\section{Background}

Lung cancer is the leading cause of cancer mortality among men and women in the United States. ${ }^{1}$ Approximately $70 \%$ of lung cancer cases are diagnosed at advanced stages when there are limited options for cure, ${ }^{2}$ reflected by the current 5 -year survival of only $18 \% .{ }^{1}$ Lowdose CT (LDCT) can detect early-stage lung cancer, increasing the likelihood of cure. The National Lung Screening Trial (NLST), a large, multicenter, US randomized controlled trial, was terminated early when it found that annual LDCT decreased the relative lung cancer-specific mortality by $20 \%$ and all-cause mortality by $6.7 \%$ compared with annual chest radiographic screening in highrisk individuals. ${ }^{3}$ The NLST defined high-risk individuals as aged 55 to 74 years, current and former smokers who quit smoking within the past 15 years, and those with at least 30 pack-years of cigarette smoking. Based on results of this trial, many professional organizations issued recommendations for LDCT, ${ }^{4-7}$ and in 2012, NCCN published the first lung cancer screening (LCS) guidelines. ${ }^{4}$ US healthcare policy decisions have also been supportive, including the US Preventive Services Task Force (USPSTF) issuance of a grade B recommendation for annual LDCT in high-risk individuals, defined as current and former smokers who have quit within the past 15 years, are aged 55 to 80 years, have at least a 30 pack-year history of cigarette smoking, and are able to undergo curative treatment. ${ }^{8}$ The Centers for Medicare \& Medicaid Services (CMS) approved LDCT coverage for a similar population of high-risk individuals, differing only in the screening end age of 77 years. ${ }^{9}$ Currently, the American Academy of Family Physicians (AAFP) is the only professional organization that does not recommend LDCT, citing insufficient evidence for or against it. ${ }^{10}$

For LDCT to be an effective population-level strategy, appropriate candidates must be identified and referred for screening. Insufficient provider knowledge of screening

See JNCCN.org for supplemental online content. 
recommendations represents a potential barrier to this process. Furthermore, providers play a key role because LDCT is currently the only cancer screening modality required by CMS to have a shared decision-making conversation for reimbursement, making it critical for providers to have knowledge of the screening recommendations, CMS coverage criteria, and evidence for screening. Early studies have shown that few providers know LCS guidelines. ${ }^{11-14}$ Studies of provider knowledge and LCS practices have been limited in several ways: (1) use of general "guideline awareness" as the definition of knowledge, (2) changes in policies that affect guideline knowledge (USPSTF and CMS), (3) a limited sample size with nondiverse provider populations, and (4) lack of adjustment for potential confounding. ${ }^{11-14}$ In this context, the present study aimed to test the hypothesis that low provider knowledge of LCS guideline recommendations would be associated with less provider-reported screening with LDCT.

\section{Patients and Methods}

\section{Study Design and Participants}

From February through May 2017, we surveyed 625 providers who routinely perform health screenings or care for patients at high risk of lung cancer, including providers who practiced within general internal medicine/ family medicine, pulmonology, hematology/oncology, and gynecology within an academic medical center (Vanderbilt University Medical Center [VUMC]) and its affiliated Veterans Health Administration (VHA), including hospital-based and community-based practices. VUMC has an American College of Radiology-accredited LCS program, and the VHA does not. Neither healthcare system had LCS-specific clinical reminders or an automated system for identifying eligible candidates. A recent Medicine Grand Rounds at VUMC focused on LCS. Eligible providers included attending physicians, physicians in training, physician assistants, and nurse practitioners who reported providing healthcare services to patients aged $>50$ in the year before the study; if respondents did not meet these criteria, the questionnaire was terminated. Providers were identified using department websites, administrative staff, and department leaders. The Institutional Review Boards at VUMC and the VHA approved the study. A waiver of documentation of written consent was granted for the study; consent was implied by clicking the questionnaire link after reviewing study information. All respondents were offered the chance to win 1 of 40 Amazon $\$ 50$ gift cards upon questionnaire completion.

\section{Study Procedures}

The questionnaire was pilot-tested by 5 providers, and minor revisions were made to use VHA-specific language. Providers were emailed the internet-based questionnaire via REDCap or VHA REDCap. ${ }^{15}$ Weekly email reminders were sent to participants who had not responded by the requested deadline, and daily reminders were sent on the final 3 days.

\section{Questionnaire Content}

\section{Independent Variable: LCS Knowledge}

Six multiple-choice items adapted from a prior study assessed provider knowledge of the USPSTF and CMS coverage criteria for LCS (see supplemental eTable 1, available with this article at JNCCN.org). ${ }^{11}$ The following items were assessed:

- Initial age of screening eligibility (correct answer: 55 years)

- Upper age limit at which a patient is no longer eligible for screening (correct answer: either 77 or 80 years)

- Minimum smoking exposure (correct answer: 30 pack-years)

- Smoking status (correct answer: current and former smokers)

- Screening frequency (correct answer: yearly)

- Screening for patients who were not surgical candidates (correct answer: no)

High LCS knowledge was defined as correctly identifying 3 major criteria to identify screening candidates: initial age of screening eligibility, minimum smoking exposure, and smoking status. Low LCS knowledge was defined as not correctly identifying these 3 criteria.

\section{Outcomes: LCS Tests}

The primary outcome was self-reported order/referral of LDCT within the past year. Secondary outcomes were selfreported ordering of nonrecommended LCS tests: chest radiograph and sputum cytology. These 3 items were adapted from the NCI National Survey of Primary Care Physicians' Cancer Screening Recommendations and Practices: Colorectal and Lung Cancer Screening Questionnaire. ${ }^{16,17}$

\section{Covariates}

Providers self-reported their sex, race/ethnicity, medical position, years in training/practice, specialty, percentage of time providing direct patient care, and practice setting (Table 1). We calculated years since terminal degree for each provider by accounting for his/her medical position and years in training for attending physicians. This was calculated separately for each specialty and categorized as $<1$ year, $1-5$ years, 6-10 years, $11-15$ years, $16-25$ years, and $>25$ years.

\section{Statistical Analysis}

The analytic sample included all providers who completed the 6 guideline knowledge items and the primary 


\section{Table 1. Characteristics of Respondents}

Total Sample $(\mathbf{N}=\mathbf{3 7 8})$

\section{Characteristic}

Sex

$n(\%)$
$214(56.6)$

$12(3.2)$

306 (81.0)

23 (6.1)

47 (12.4)

7 (1.9)

Other

Medical position

Attending

Physician-in-training

178 (47.1)

161 (42.6)

39 (10.3)

Nurse practitioner/physician assistant

Years since completion of terminal degree

\begin{tabular}{lc}
$<1 y$ & $39(10.3)$ \\
\hline $1-5 y$ & $139(36.8)$ \\
\hline $6-15 y$ & $106(28.0)$ \\
\hline $16-25 y$ & $57(15.1)$ \\
$>25 y$ & $34(9.0)$ \\
\hline Missing & $3(0.8)$
\end{tabular}

Primary field of practice

General internal medicine

Internal medicine, pulmonology

174 (46.0)

26 (6.9)

39 (10.3)

Internal medicine, hematology/oncology

Family medicine

$73(19.3)$

12 (3.2)

Gynecology

48 (12 0.7)

Other

$6(1.6)$

$289(76.5)$

271 (71.7)

107 (28.3)

$285(75.4)$

$93(24.6)$

$25(15-40)$

Perceived percentage of current smokers in

providers' practices, median (IQR)

Perceived percentage of former smokers in

providers' practices, median (IQR)

Percentage of time providing direct patient care

Community-based

$30(20-50)$

system

\begin{tabular}{lrrr}
\hline$\leq 50 \%$ & $89(23.5)$ & $44(30.3)$ & $45(19.3)$ \\
\hline$>50 \%$ & $289(76.5)$ & $101(69.7)$
\end{tabular}

High LCS Knowledge ${ }^{a}(N=145)$

n (\%)

$75(51.7)$

139 (59.7)

$5(3.4)$

7 (3.0)

114 (78.6)

192 (82.4)

9 (6.2)

14 (6.0)

21 (14.5)

26 (11.2)

3 (2.1)

4 (1.7)

66 (45.5)

112 (48.1)

73 (50.3)

88 (37.8)

6 (4.1)

33 (14.2)

27 (11.6)

68 (46.9)

71 (30.5)

35 (24.1)

71 (30.5)

19 (13.1)

38 (16.3)

$10(6.9)$

24 (10.3)

86 (59.3)

88 (37.8)

10 (6.9)

16 (6.9)

19 (13.1)

20 (8.6)

23 (15.9)

50 (21.5)

4 (2.8)

8 (3.4)

2 (1.4)

46 (19.7)

1 (0.7)

5 (2.1)

112 (77.2)

159 (68.2)

33 (22.8)

74 (31.8)

Abbreviations: CMS, Centers for Medicare \& Medicaid Services; IQR, interquartile range; LCS, lung cancer screening; USPSTF, US Preventive Services Task Force; VHA, Veterans Health Administration.

aDefined as correctly identifying USPSTF/CMS screening eligibility start age as 55 years, smoking status of current or former smoker, and smoking history of $\geq 30$ packyears.

befined as not identifying USPSTF/CMS screening eligibility start age as 55 years, smoking status of both current or former smoker, and smoking history of $\geq 30$ packyears. 
outcome. Descriptive statistics were used to characterize provider characteristics.

Multivariable logistic regression was used to assess the association between knowledge and LDCT order/referral, with adjustment for the following covariates: specialty, years since terminal degree, and healthcare system. Adjusted odds ratios (aORs) and 95\% CIs were reported. All analyses were conducted using R software (R Foundation for Statistical Computing).

\section{Sensitivity and Subgroup Analyses}

We tested the sensitivity of our definition of guideline knowledge through several analyses. First, we redefined low knowledge as answering less than any 3 of 6 guideline items correctly. Next, we converted knowledge into a continuous variable. For this variable, we defined knowledge based on scores $(0=$ least knowledge; $6=$ most knowledge). We determined that individual guideline components were most strongly associated with LDCT order/referral. These knowledge components in combination with the highest number of correctly identified guideline components were used to develop the fourth definition of knowledge. In addition, because we evaluated knowledge and practice at different hospitals and between academic and community practices, we analyzed for effect modification among these subgroups.

\section{Results}

We invited 625 providers (449 academic, 176 VHA) to complete the survey, and 407 (65\%) responded (309 academic, 98 VHA). We excluded 10 participants who did not meet eligibility criteria and another 4 respondents with duplicate responses because of a dual academic-VHA appointment; all 4 work predominantly at VHA and were reclassified as such. A total of 15 questionnaires were missing responses to the knowledge items or the primary outcome variable, yielding an analytic sample of 378 (60.5\%) (Figure 1).

\section{Characteristics of Respondents}

Questionnaire nonresponders, compared with responders, were more likely to be attending physicians ( $61 \%$ vs $47.1 \% ; P=.001)$ and to work at the VHA $(35.8 \%$ vs $24.6 \% ; P=.004$ ) (supplemental eTable 2). Of the respondents, $233(62 \%)$ had low LCS knowledge (Table 1 and supplemental eFigure 1). Compared with providers with high LCS knowledge, more providers with low LCS knowledge were female $(59.7 \%$ vs $51.7 \%)$, were advanced practice practitioners ( $14.2 \%$ vs $4.1 \%)$, were within 6 to 15 years of their terminal degree $(30.5 \%$ vs $24.1 \%)$, and practiced in gynecology ( $19.7 \%$ vs $1.4 \%)$, in

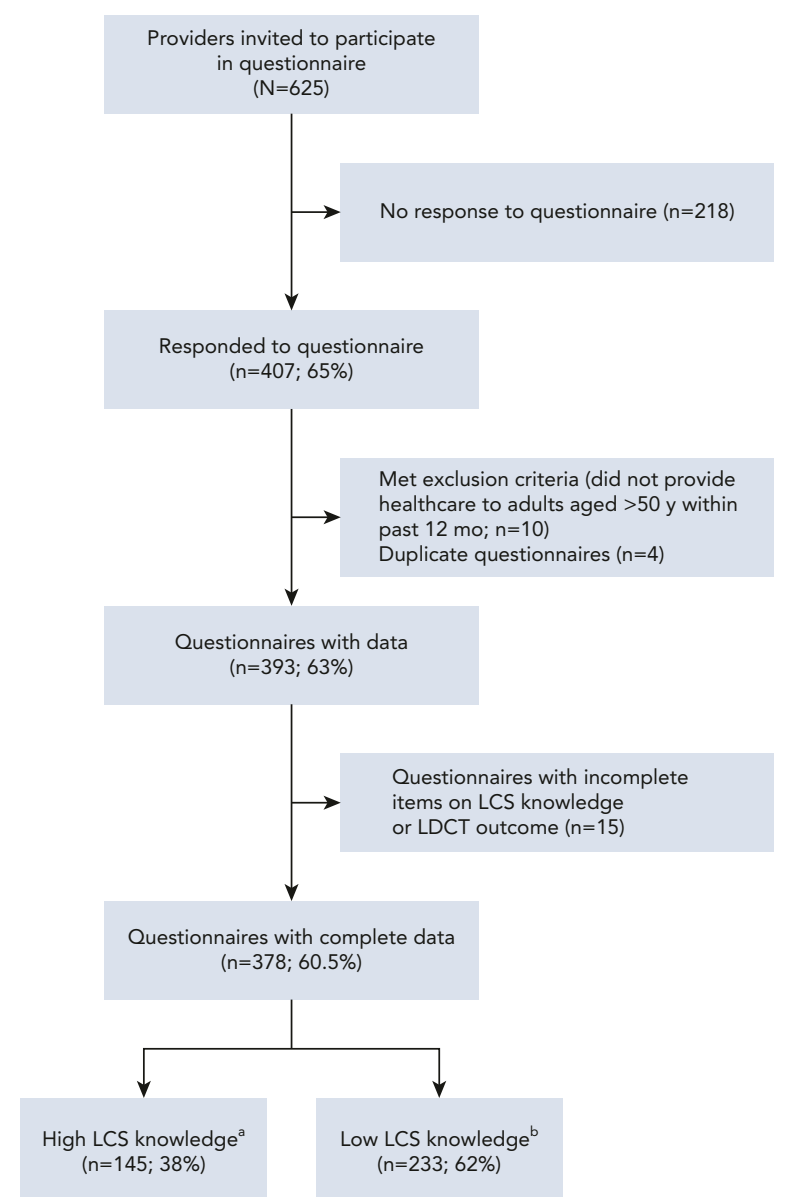

Figure 1. CONSORT diagram.

Abbreviations: CMS, Centers for Medicare \& Medicaid Services; LCS, lung cancer screening; LDCT, low-dose CT; USPSTF, US Preventive Services Task Force.

aDefined as correctly identifying USPSTF/CMS screening eligibility start age as 55 years, smoking status of current or former smoker, and smoking history of $\geq 30$ pack-years.

${ }^{b}$ Defined as not identifying USPSTF/CMS screening eligibility start age as 55 years, smoking status of both current or former smoker, and smoking history of $\geq 30$ pack-years.

a community-based practice $(31.8 \%$ vs $22.8 \%)$, or at an academic medical center $(78.5 \%$ vs $70.3 \%)$ (Table 1$)$.

\section{Primary Outcome: LDCT Screening and LCS Knowledge}

A total of 224 providers (59\%) reported ordering/referring for LDCT within the past year. The unadjusted odds of ordering/referring for LDCT were $76 \%$ lower for providers with low LCS knowledge than for those with high LCS knowledge (Table 2). Results were attenuated but remained robust to adjustment for primary field of practice, years since completion of terminal degree, and healthcare system (aOR, 0.41; 95\% CI, 0.24-0.71).

Rates of ordering/referring for LDCT were highest among general internal medicine/primary care providers 


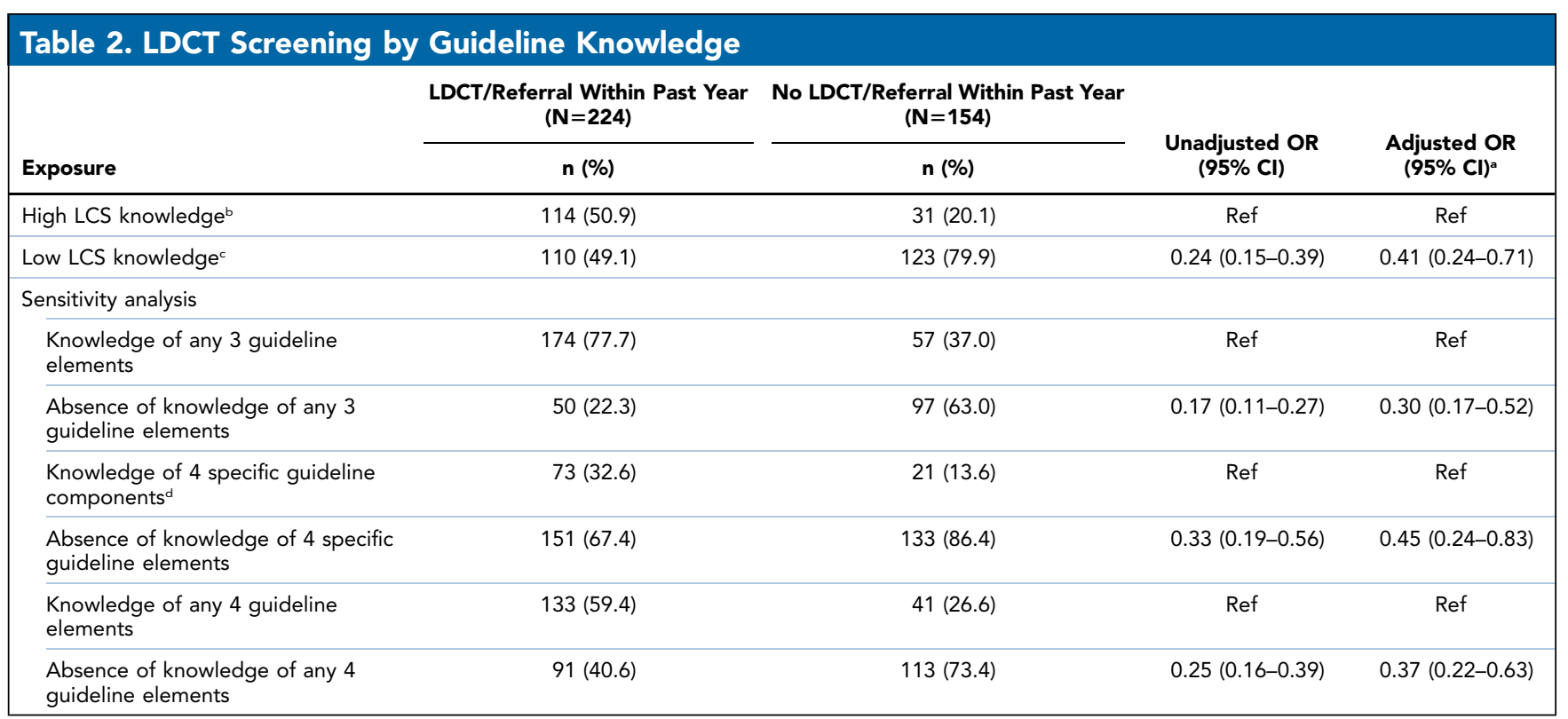

Abbreviations: CMS, Centers for Medicare \& Medicaid Services; LCS, lung cancer screening; LDCT, low-dose CT; OR, odds ratio; USPSTF, US Preventive Services Task Force.

${ }^{a}$ Covariates adjusted in model: specialty, years in practice, healthcare system.

bDefined as correctly identifying USPSTF/CMS screening eligibility start age as 55 years, smoking status of current or former smoker, and smoking history of $\geq 30$ packyears.

'Defined as not identifying USPSTF/CMS screening eligibility start age as 55 years, smoking status of current or former smoker, and smoking history of $\geq 30$ packyears.

dThe 4 guideline items were identified by logistic regression and included screening eligibility start age as 55 years, smoking status of current or former smoker, screening interval as annual, and screening not recommended for patients unable to undergo surgery.

(75.9\%), followed by pulmonologists $(74.4 \%)$, hematologists/ oncologists $(39.7 \%)$, and gynecologists $(2.1 \%)$. After adjustment for other factors, all provider types were less likely than general internists/primary care providers to order/refer for LDCT: gynecology (aOR, 0.01; 95\% CI, 0.001-0.07), hematology/oncology (aOR, 0.17; 95\% CI, $0.09-0.31$ ), and pulmonology providers (aOR, 0.77; 95\% CI, 0.32-1.82).

\section{Secondary Outcomes: Chest Radiograph and Sputum Cytology}

A total of 74 respondents reported ordering chest radiographs and 12 reported ordering sputum cytology within the past year. After adjustment for specialty, years since terminal degree, and healthcare system, providers with low LCS knowledge had a 2.7 higher odds of ordering a screening chest radiograph than providers with high LCS knowledge (95\% CI, 1.4-5.0). Numbers of self-reported sputum cytology were too small to test differences between those with high and low LCS knowledge.

\section{Sensitivity and Subgroup Analyses}

When redefining low LCS knowledge as correctly identifying $<3$ guideline components, the aOR of selfreported LDCT order/referral within the past year among providers with low versus high knowledge was 0.30
(95\% CI, 0.17-0.52). The distribution of correctly answered guideline knowledge items is shown in supplemental eFigure 1. Assessment of guideline knowledge as a continuous variable showed a statistically significant positive linear relationship between the number of correctly identified LCS guideline elements for scores 0 through 4 and LDCT orders/referrals (Figure 2). The aOR for LDCT ordering/referral decreased for scores of 5 and 6 and

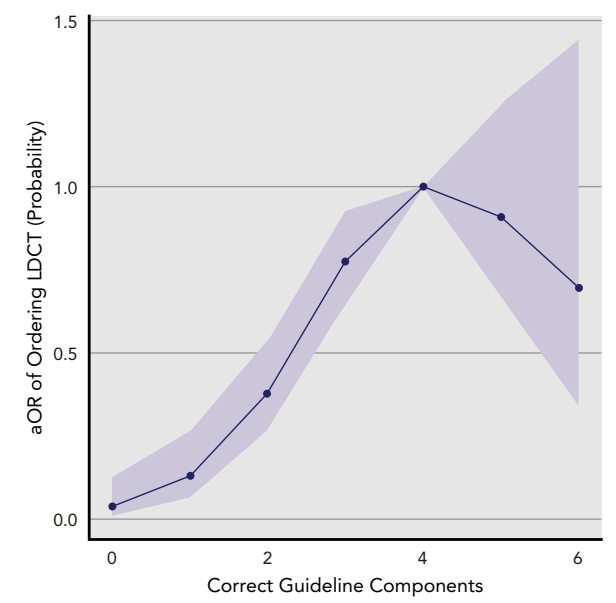

Figure 2. LDCT screening by knowledge as a continuous variable. Abbreviations: aOR, odds ratio; LDCT, low-dose CT. 
was not statistically significant. When low LCS knowledge was defined as correctly identifying $<4$ LCS guideline elements, the aOR of LDCT order/referral among high versus low knowledge was 0.37 (95\% CI, 0.22-0.63). In the adjusted model, the 4 guideline components most strongly associated with LDCT order/referral were start age of 55 years, smoking status as both current and former, screening interval as annual, and screening not recommended for patients unable to undergo surgery. Incorrect responses to these 4 specific items were associated with a lower odds of LDCT screening (aOR, $0.45 ; 95 \%$ CI, 0.24-0.83) (Table 2). There was no evidence of effect modification by healthcare system or practice location (Table 3 and supplemental eFigure 2).

\section{Discussion}

Results of this study represent the strongest evidence to date of the independent relationship between guideline knowledge and evidence-based LCS. Novel in our methodology, we found that providers with low guideline knowledge were less likely to perform LDCT lung screening. This association was robust to a sensitivity analysis and was not modified by healthcare system or practice location. This study extends prior knowledge by testing this relationship after issuance of US policy decisions supporting LDCT screening in a large sample of multiple provider types, clinical settings, and across
2 healthcare systems with an excellent response rate, thereby overcoming many of the limitations of prior research in this area. ${ }^{11,12,14,18}$

One of our key findings is that LCS guideline knowledge is currently low. Prior studies have described low awareness or knowledge of LCS guidelines. ${ }^{11-13,18-23} \mathrm{~A}$ survey conducted in 2013 found that only $47 \%$ of primary care providers at an academic medical center knew at least 3 of 6 LCS guideline items. Similarly, a 2015 survey found that most South Carolina family physicians were not aware of their own organization's (AAFP) position on LCS, ${ }^{13}$ and a more recent study found that only $10 \%$ of primary care providers and pulmonologists were able to identify all of the CMS elements needed for documentation..$^{20}$

Most providers in the present study reported ordering/referring a patient for LDCT screening within the past year. Fortunately, rates of referral have increased since $2013\left(12 \%\right.$ vs 59\%), ${ }^{11}$ a trend that may be explained by the 2014 USPSTF grade B recommendation and CMS's 2015 decision to cover LDCT lung screening. ${ }^{8,9}$ An increased rate of LCS referral since 2013 is consistent with a recent national study in which $36 \%$ of providers reported ordering 1 to 5 tests in the past year. ${ }^{24}$ However, $<1 \%$ of gynecologists and only 39\% of hematology/oncology providers reported performing LDCT screening in the past year in our study. Having a patient population that is ineligible for screening (ie, younger nonsmokers) or

\section{Table 3. Effect Modification by Healthcare System and Practice Location}

\begin{tabular}{|c|c|c|c|c|}
\hline Exposure & LDCT/Referral Within Past Year & No LDCT/Referral Within Past Year & $\begin{array}{c}\text { Unadjusted OR } \\
(95 \% \mathrm{CI})\end{array}$ & $\begin{array}{l}\text { Adjusted OR } \\
(95 \% \mathrm{Cl})\end{array}$ \\
\hline \multicolumn{5}{|l|}{ Healthcare system } \\
\hline High LCS knowledge ${ }^{\mathrm{b}}$ & 39 (41.9) & $4(4.3)$ & Ref & Ref \\
\hline Low LCS knowledge ${ }^{c}$ & $34(36.6)$ & $16(17.2)$ & $0.22(0.07-0.72)$ & $0.25(0.06-1.03)$ \\
\hline Low LCS knowledge ${ }^{c}$ & $76(36.1)$ & $107(37.5)$ & $0.26(0.15-0.43)$ & $0.48(0.25-0.92)$ \\
\hline \multicolumn{5}{|l|}{ Practice location } \\
\hline \multicolumn{5}{|c|}{ Hospital-based clinic ${ }^{d}(n=271)$} \\
\hline High LCS knowledge ${ }^{\mathrm{b}}$ & $88(32.4)$ & $24(8.9)$ & Ref & Ref \\
\hline \multicolumn{5}{|c|}{ Community-based clinic ${ }^{d}(n=107)$} \\
\hline Low LCS knowledge ${ }^{c}$ & $32(29.9)$ & $42(39.3)$ & $0.21(0.08-0.53)$ & $0.29(0.08-1.10)$ \\
\hline
\end{tabular}

Abbreviations: CMS, Centers for Medicare \& Medicaid Services; LCS, lung cancer screening; LDCT, low-dose computed tomography; OR, odds ratio; USPSTF, U.S. Preventive Services Task Force; VHA, Veterans Health Administration.

aCovariates adjusted in model: specialty and years in practice.

bDefined as correctly identifying USPSTF/CMS screening eligibility start age as 55 years, smoking status of current or former smoker, and smoking history of $\geq 30$ packyears.

'Defined as not identifying USPSTF/CMS screening eligibility start age as 55 years, smoking status of current or former smoker, and smoking history of $\geq 30$ packyears.

${ }^{\mathrm{d} C}$ Covariates adjusted in model: specialty, years since terminal degree, and healthcare system. 
assuming that another provider is addressing cancer screening may explain lower knowledge or rates of screening among these providers.

The lower screening rate observed among pulmonologists is somewhat surprising. The belief that the primary care provider should be responsible for cancer screenings may be one explanation..$^{20}$ Ambivalence among pulmonologists regarding the utility of screening may have contributed to the lower screening rate, but this may resolve as new data increasingly support LCS to reduce mortality ${ }^{18,25}$ Finally, selection bias may also be present. This finding certainly warrants further study. Overall, our results suggest that future educational interventions should broadly target primary care and specialty providers who care for patients at high risk for lung cancer.

Consistent with prior studies, many providers still report ordering screening chest radiographs, which is not a recommended screening test. ${ }^{4,11,13,19,21,24}$ It is not surprising that there were more providers with low LCS knowledge who reported ordering screening chest radiographs. However, to our knowledge, this is the first study showing this relationship, and this finding further strengthens the relationship between guideline knowledge and LCS. It also highlights the need to emphasize the inappropriate use of chest radiography for screening and to assess its use in future studies.

Multiple factors may explain why providers may or may not adhere to LCS guidelines. ${ }^{26}$ Because LDCT is a relatively new test, many providers may not be familiar with or aware of the guidelines. ${ }^{26}$ In addition, the degree to which guidelines differ may be confusing providers, thus affecting attitudes and screening behavior, is unclear, but is an important factor to consider. ${ }^{26}$ Provider education on guideline recommendations and the efficacy of screening is crucial, especially in the early stages of implementing a new tool. However, education alone is unlikely to be successful in improving screening behavior due to multiple factors that influence these decisions. ${ }^{26}$

The complexity of LCS guidelines makes changes to healthcare processes critical for effective, efficient screening. ${ }^{4}$ Through system-level changes, guideline knowledge could be taught or reinforced through clinical reminders or decision support programs. Electronic medical records (EMRs) could be leveraged to identify eligible patients and notify providers of the need to address screening. Unfortunately, current EMR systems are not adequately capturing smoking history, which limits this approach. In the VHA LCS demonstration project, approximately $40 \%$ of patients who met initial life expectancy, comorbidity, and age criteria either were missing information in the EMR on smoking status or had tobacco pack-years incorrectly calculated. ${ }^{27} \mathrm{~A}$ recent study found a $96.2 \%$ discordance between smoking history from the EMR compared with the history elicited from shared decision-making, and $53.6 \%$ of eligible individuals would have failed to meet screening criteria if the EMR were solely relied on to identify eligible screening candidates. ${ }^{28}$ Finally, eligibility criteria should be made easily accessible in familiar, convenient, and diverse locations (eg, internet-based resources, hand-held electronic applications, posters) to maximally support providers across healthcare settings. The NCCN Clinical Practice Guidelines in Oncology for LCS are available as an internetbased resource for providers to use free of charge. ${ }^{4}$

Even as healthcare system processes adapt over time, providers will remain key to LCS. Lung cancer is the only cancer screening that requires a provider to conduct a shared decision-making visit for CMS reimbursement. ${ }^{9}$ Furthermore, provider recommendation is a predictor of patient behavior. ${ }^{29}$ In one study, $82 \%$ of patients reported that they would undergo LDCT lung screening if recommended by their physician. ${ }^{30}$ Another study found that approximately $85 \%$ of LCSadherent patients reported "trust in their provider" as a reason for undergoing screening. ${ }^{12}$ Finally, referral for LCS should be shared among providers (eg, primary care, pulmonology, oncology), with the common goal of maximizing screening among eligible candidates.

The overall response rate of $65 \%$ is on the higher end of the published range $(9 \%-94 \%)$ for internetbased surveys of healthcare providers. ${ }^{31}$ However, our study has several limitations. Selection bias is a possible consequence of a $<100 \%$ response rate. In addition, we relied on provider self-reporting of screening practice, which could be subject to social desirability bias and recall bias. Furthermore, our definition of guideline knowledge did not include years since quitting for former smokers ( $\leq 15$ years), because we elected to focus on guideline elements that providers would likely recognize, such as smoking status (current or former smoker) and pack-years of exposure $(\geq 30)$. Results of this survey may also not be generalizable. Finally, our study was not designed to elicit why providers do or do not perform LDCT screening. Future directions should include qualitative work to better understand this population's barriers and facilitators to performing LCS.

\section{Conclusions}

Findings of this cross-sectional study showed that provider LCS guideline knowledge is a strong and independent predictor of LCS practices. Provider knowledge of the USPSTF guidelines and CMS coverage criteria was also found to be significantly low. Provider educational and healthcare system interventions are needed to facilitate the appropriate identification of candidates for LCS to be effective at the population level. 


\section{Acknowledgments}

The authors wish to thank the survey participants. The authors also wish to acknowledge Norman Chandler Hardman, Dave Dobrzynski, and Sade Arinze for participation in the survey pilot, and Pamela Watts, Michael Beasley, Ashley Haston, and Ted Anderson for their assistance with the survey recruitment.

Submitted July 2, 2018; accepted for publication October 22, 2018.

Author contributions: Study concept and design: Lewis, Weaver, Sandler, Horn, Massion, Tindle. Data curation and investigation: Lewis, Chen, Horn,
Massion, Tindle. Data analysis and interpretation: Lewis, Chen, Weaver, Massion, Roumie, Tindle. Manuscript preparation: All authors. Final approval of manuscript: All authors.

Disclosures: The authors have not received any financial consideration from any person or organization to support the preparation, analysis, results, or discussion of this article.

Funding: This material is based on work supported by the Office of Academic Affiliations, VA National Quality Scholars Program, with resources and use of facilities at Veterans Health Administration - Tennessee Valley Healthcare System, Nashville, Tennessee.

Correspondence: Jennifer A. Lewis, MD, MS, Division of Hematology/ Oncology, Department of Medicine, Vanderbilt University Medical Center, 2220 Pierce Avenue, Nashville, TN 37232-6307.

Email: jennifer.a.lewis@vanderbilt.edu

\section{References}

1. American Cancer Society. Cancer facts \& figures. Atlanta, GA: American Cancer Society; 2018

2. Morgensztern $\mathrm{D}, \mathrm{Ng} \mathrm{SH}, \mathrm{Gao} F$, et al. Trends in stage distribution for patients with non-small cell lung cancer: a National Cancer Database survey. J Thorac Oncol 2010;5:29-33.

3. National Lung Screening Trial Research Team. Reduced lung-cancer mortality with low-dose computed tomographic screening. N Engl J Med 2011;365:395-409.

4. Wood DE, Kazerooni EA, Baum SL, et al. NCCN Clinical Practices Guidelines in Oncology: Lung Cancer Screening. Version 2.2019. Accessed September 18, 2018. To view the most recent version of these guidelines, visit NCCN.org.

5. Wender R, Fontham ET, Barrera E Jr, et al. American Cancer Society lung cancer screening guidelines. CA Cancer J Clin 2013;63:106-117.

6. Jaklitsch MT, Jacobson FL, Austin JH, et al. The American Association for Thoracic Surgery guidelines for lung cancer screening using low-dose computed tomography scans for lung cancer survivors and other high-risk groups. J Thorac Cardiovasc Surg 2012; 144:33-38.

7. Bach PB, Mirkin JN, Oliver TK, et al. Benefits and harms of CT screening for lung cancer: a systematic review. JAMA 2012;307:2418-2429.

8. Moyer VA; U.S. Preventive Services Task Force. Screening for lung cancer: U.S. Preventive Services Task Force recommendation statement. Ann Intern Med 2014;160:330-338.

9. Decision memo for screening for lung cancer with low dose computed tomography (LDCT) (CAG-00439N). Centers for Medicare \& Medicaid Services website. Available at: https://www.cms.gov/medicare-coveragedatabase/details/nca-decision-memo.aspx?NCAld=274. Accessed January 4, 2018.

10. Clinical preventive service recommendation: lung cancer. American Academy of Family Physicians website. Available at: https://www.aafp. org/patient-care/clinical-recommendations/all/lung-cancer.html. Accessed September 18, 2018.

11. Lewis JA, Petty WJ, Tooze JA, et al. Low-dose CT lung cancer screening practices and attitudes among primary care providers at an academic medical center. Cancer Epidemiol Biomarkers Prev 2015;24:664-670.

12. Duong DK, Shariff-Marco S, Cheng I, et al. Patient and primary care provider attitudes and adherence towards lung cancer screening at an academic medical center. Prev Med Rep 2017;6:17-22.

13. Ersek JL, Eberth JM, McDonnell KK, et al. Knowledge of, attitudes toward, and use of low-dose computed tomography for lung cancer screening among family physicians. Cancer 2016;122:2324-2331.

14. Raz DJ, Wu GX, Consunji M, et al. The effect of primary care physician knowledge of lung cancer screening guidelines on perceptions and utilization of low-dose computed tomography. Clin Lung Cancer 2018;19:51-57.

15. Harris PA, Taylor R, Thielke R, et al. Research electronic data capture (REDCap)—a metadata-driven methodology and workflow process for providing translational research informatics support. J Biomed Inform 2009;42:377-381.

16. National Survey of Primary Care Physicians' Cancer Screening Recommendations and Practices: Colorectal and Lung Cancer Screening
Questionnaire. Published March 21, 2006. Available at: https:// healthcaredelivery.cancer.gov/screening_rp/screening_rp_colo_lung_ inst.pdf?file=/surveys/screening_rp/screening_rp_colo_lung_inst.pdf. Accessed March 21, 2006

17. Klabunde CN, Marcus PM, Han PK, et al. Lung cancer screening practices of primary care physicians: results from a national survey. Ann Fam Med 2012;10:102-110.

18. laccarino JM, Clark J, Bolton R, et al. A national survey of pulmonologists views on low-dose computed tomography screening for lung cancer. Ann Am Thorac Soc 2015;12:1667-1675.

19. Hoffman RM, Sussman AL, Getrich CM, et al. Attitudes and beliefs of primary care providers in New Mexico about lung cancer screening using low-dose computed tomography. Prev Chronic Dis 2015;12:150112.

20. Triplette $M$, Kross EK, Mann BA, et al. An assessment of primary care and pulmonary provider perspectives on lung cancer screening. Ann Am Thorac Soc 2018;15:69-75.

21. Raz DJ, Wu GX, Consunji $M$, et al. Perceptions and utilization of lung cancer screening among primary care physicians. J Thorac Oncol 2016; 11:1856-1862.

22. Simmons VN, Gray JE, Schabath MB, et al. High-risk community and primary care providers knowledge about and barriers to low-dose computed topography lung cancer screening. Lung Cancer 2017;106: 42-49.

23. Kanodra NM, Pope $\mathrm{C}$, Halbert $\mathrm{CH}$, et al. Primary care provider and patient perspectives on lung cancer screening: a qualitative study. Ann Am Thorac Soc 2016;13:1977-1982.

24. Eberth JM, McDonnell KK, Sercy E, et al. A national survey of primary care physicians: perceptions and practices of low-dose CT lung cancer screening. Prev Med Rep 2018;11:93-99.

25. De Koning H, Van Der Aalst C, Ten Haaf K, et al. Effects of volume CT lung cancer screening: mortality results of the NELSON randomised-controlled population based trial. Presented at the International Association for the Study of Lung Cancer 19th World Conference on Lung Cancer; September 25, 2018; Toronto, Ontario, Canada. Abstract PL02.05

26. Cabana MD, Rand CS, Powe NR, et al. Why don't physicians follow clinical practice guidelines? A framework for improvement. JAMA 1999;282: 1458-1465.

27. Kinsinger LS, Anderson C, Kim J, et al. Implementation of lung cancer screening in the Veterans Health Administration. JAMA Intern Med 2017, 177:399-406.

28. Modin HE, Fathi JT, Gilbert CR, et al. Pack-year cigarette smoking history for determination of lung cancer screening eligibility: comparison of the electronic medical record versus a shared decision-making conversation. Ann Am Thorac Soc 2017;14:1320-1325.

29. Zapka JG, Lemon SC. Interventions for patients, providers, and health care organizations. Cancer 2004;101(5 Suppl):1165-1187.

30. Jonnalagadda S, Bergamo C, Lin JJ, et al. Beliefs and attitudes about lung cancer screening among smokers. Lung Cancer 2012;77:526-531.

31. Braithwaite D, Emery J, De Lusignan S, et al. Using the Internet to conduct surveys of health professionals: a valid alternative? Fam Pract 2003;20: 545-551. 